\title{
Mobility of signaling molecules: the key to deciphering plant organogenesis
}

\author{
Kensuke Kawade $\cdot$ Hirokazu Tanimoto
}

Received: 1 July 2014 / Accepted: 25 November 2014 / Published online: 17 December 2014

(C) The Botanical Society of Japan and Springer Japan 2014

\begin{abstract}
Signaling molecules move between cells to form a characteristic distribution pattern within a developing organ; thereafter, they spatiotemporally regulate organ development. A key question in this process is how the signaling molecules robustly form the precise distribution on a tissue scale in a reproducible manner. Despite of an increasing number of quantitative studies regarding the mobility of signaling molecules, the detail mechanism of organogenesis via intercellular signaling is still unclear. We here review the potential advantages of plant development to address this question, focusing on the cytoplasmic continuity of plant cells through the plasmodesmata. The plant system would provide a unique opportunity to define the simple transportation mode of diffusion process, and, hence, the mechanism of organogenesis via intercellular signaling. Based on the advances in the understanding of intercellular signaling at the molecular level and in the quantitative imaging techniques, we discuss our current challenges in measuring the mobility of signaling molecules for deciphering plant organogenesis.
\end{abstract}

Keywords Intercellular signaling · Morphogen gradient · Plant organogenesis · Protein diffusion dynamics

\footnotetext{
K. Kawade $(\bowtie)$

Department of Biological Sciences, Faculty of Science, Hokkaido University, Kita 10 Nishi 8, Kita-ku, Sapporo 060-0810, Japan e-mail: kawa-ken@mail.sci.hokudai.ac.jp

K. Kawade

RIKEN Center for Sustainable Resource Science,

1-7-22 Suehiro-cho, Tsurumi-ku, Yokohama,

Kanagawa 230-0045, Japan

H. Tanimoto

Institut Jacques Monod, UMR 7592 CNRS, 15 rue Hélène Brion,
} 75205 Paris, France

\section{Introduction}

A developing organ comprises a heterogeneous field of individual cells that differ in terms of physical conditions, molecular constitution, structures, differentiation stages and other properties. Cells orchestrate such variables using cell-to-cell communication to achieve normal organogenesis. The movement of signaling molecules between cells defines their spatiotemporal distribution within a developing organ, and, thereafter, guides developmental progression. Thus, quantitative characterization of the movement of signaling molecules would facilitate understanding of the mechanism of organogenesis. The kinetics of intercellular signaling is being investigated in animal development, using quantitative imaging techniques such as fluorescence redistribution (or recovery) after photobleaching (FRAP) and photoconversion of fluorescent proteins (reviewed in Kicheva et al. 2012; Müller et al. 2013; Wartlick et al. 2009). Pioneer studies have reported that signaling molecules are transported in a non-directed manner and that their tissue-scale distribution can be explained, at least in part, by the kinetics of their movement (Kicheva et al. 2007; Müller et al. 2012; Yu et al. 2009).

The movement of signaling molecules between cells is essential for normal organogenesis in plants, as well as in animals. We showed recently that the transcriptional co-activator ANGUSTIFOLIA3 (AN3, which is also known as GRF-INTERACTING FACTOR1) (Horiguchi et al. 2005; Kim and Kende 2004) is produced within mesophyll cells of leaf primordia in Arabidopsis thaliana (hereafter, Arabidopsis), and then moves into epidermal cells (Kawade et al. 2013). Interference with AN3 movement causes defective proliferation of epidermal cells, resulting in abnormal leaf size and shape (Kawade et al. 2013). A series of studies have identified various signaling 
molecules including AN3, and revealed their functional significance in plant organogenesis (e.g. Jackson et al. 1994; Kawade et al. 2013; Kim et al. 2003; Nakajima et al. 2001). However, our quantitative understanding of the spatiotemporal dynamics of intercellular signaling in plants is limited.

This review aims to highlight the importance of quantitative investigation of signaling molecule mobility to increase our knowledge of plant organogenesis. Quantitative analysis of the movement of signaling molecules in plants can be a promising target because of the simple transportation mode; i.e. via the plasmodesmata. Also, comparison of quantitative information of intercellular signaling between animals and plants would enhance our understanding of the similarities and differences in their development. We will firstly see how the tissue-scale distribution of signaling molecules is generated by their intercellular movement using a simple mathematical model. We will next describe the features of intercellular signaling in plants, focusing on plasmodesmata to emphasize the advantages of plant development systems for investigation of the dynamics of intercellular signaling. Lastly, given the advances in the molecular understanding of plant development including AN3 signaling, we will discuss current challenges in quantitative assessment of signaling molecule dynamics during plant organogenesis.

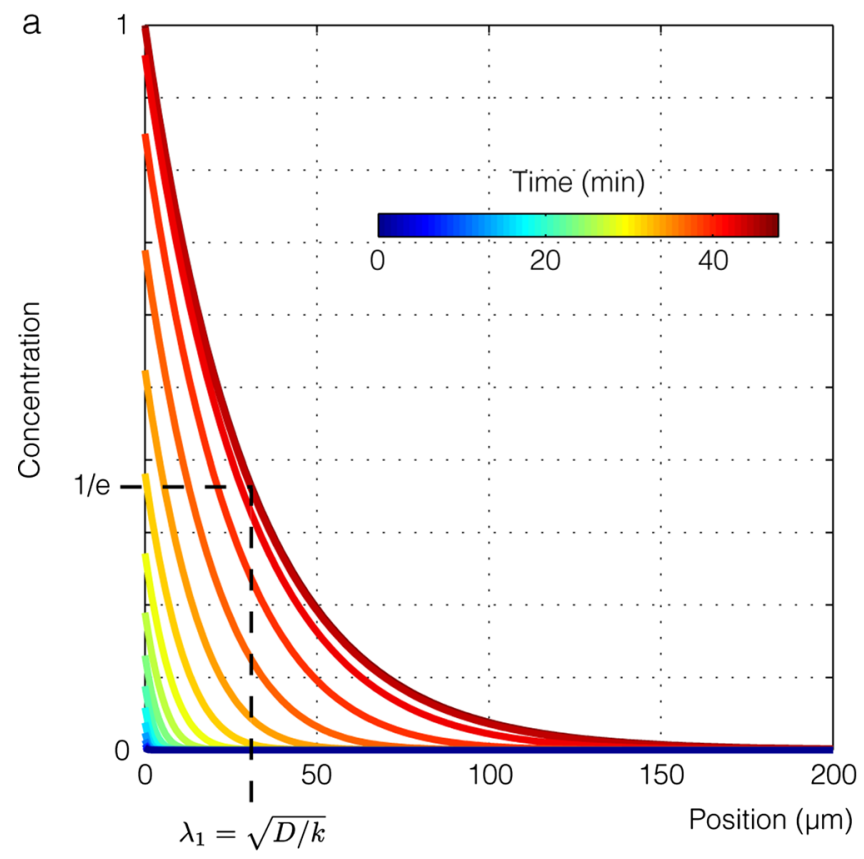

Fig. 1 Gradient formation in the SDD model. a Time-evolution of distribution of signaling molecules is depicted using the SDD model. Equation 1 is numerically solved with parameters of $D=3 \mu \mathrm{m}^{2} \mathrm{~s}^{-1}$ and $k_{\mathrm{d}}=3.2 \times 10^{-3}$, which yields $\lambda=30 \mu \mathrm{m}$. Colours indicate dif-
Tissue-scale distribution of signaling molecules via diffusion process

Diffusion of signaling molecules has long been proposed as a primary mechanism of establishment of a tissue-scale gradient distribution (Crick 1970; Turing 1952; Wolpert 1969). Quantitative studies revealed the contribution of diffusion process for morphogen gradient formation in animal development (reviewed in Mülle et al. 2013). Mathematically, diffusion is defined as a process in which movement of materials in a given time is proportional to the concentration difference between two neighbor positions (Berg 1993). The simplest example is random motion of small particles in water (i.e. Brownian motion, Brown 1828), which is passively driven by thermal fluctuations of water molecules. Movement of signaling molecules in biological processes through transcytosis and facilitated or suppressed transportation through interaction with other molecules can also be described as diffusion if it satisfies the above conditions.

To assess the mechanism by which signaling molecules form a tissue-scale gradient distribution through the diffusion process, let us consider a simple one-dimensional mathematical model [Synthesis, Diffusion and Degradation (SDD) model (Crick 1970), Fig. 1]. Signaling molecules are constantly produced at a boundary of a tissue $(x=0)$.

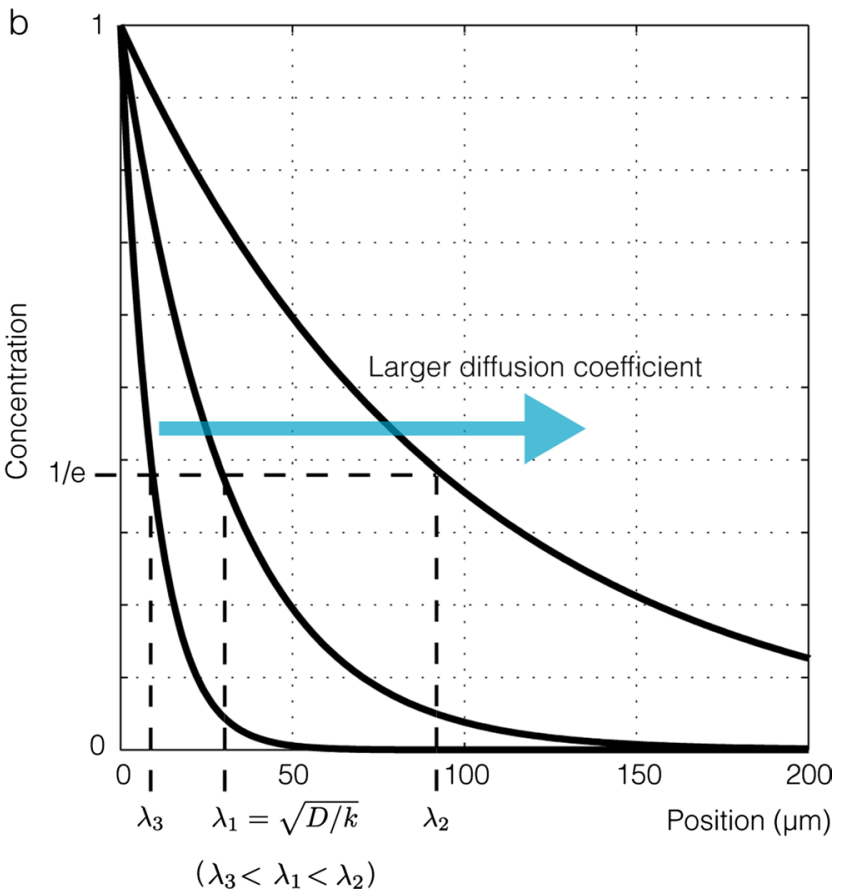

ferent time points. b Relationship between gradient pattern and diffusion coefficient. Molecules that more rapidly form a broader distribution. $D=0.3,3$ and $30 \mu \mathrm{m}^{2} \mathrm{~s}^{-1}$ (left to right) 
They then spread into the entire tissue $(x>0)$ by diffusion in association with constant degradation. These three processes can be modeled by a reaction-diffusion equation,

$\frac{\partial C(x, t)}{\partial t}=D \frac{\partial^{2} C(x, t)}{\partial x^{2}}-k_{d} C(x, t)$.

$C(x, t)$ denotes the concentration of signaling molecule at position $x$ and time $t$. The first term at the right hand side is the transportation of signaling molecules by diffusion, where $D$ is a diffusion coefficient (unit: length ${ }^{2}$ time $^{-1}$ ). The second term is the spatially uniform degradation of signaling molecules with a degradation rate $k_{\mathrm{d}}\left(\mathrm{time}^{-1}\right)$. This equation should be complemented by two boundary conditions at $x=0$ and $x=\infty$. The first condition is determined by the assumption that signaling molecules are constantly produced at the left boundary $(x=0)$ with a rate $J$. This condition is expressed as

$\left.\frac{\partial C(x, t)}{\partial x}\right|_{x=0}=-\frac{J}{D}$,

where the production $J$ is balanced by the diffusive flux $-D \partial_{x} C$. For the second boundary condition at $x=\infty$, we assume that the tissue is so large that it can be approximated as a semi-infinite system. The second condition is expressed as

$\left.\frac{\partial C(x, t)}{\partial x}\right|_{x=\infty}=0$.

Equations 1-3 describe how the concentration of signaling molecules $C$ changes over time (Berezhkovskii et al. 2010; Bergmann et al. 2007). Since there is no signaling molecule at $t=0, C(x, t=0)=0$ for any $x$ (Fig. 1a, blue line). Signaling molecules are produced and move into an entire tissue at $t>0$, and then approach to a steady state (from blue to red lines in Fig. 1a) where the concentration does not change in time as $\partial_{t} C(x, t)=0$. This final profile $\tilde{C}(x, t)$ can be therefore obtained by setting Eq. $1=0$,

$D \frac{d^{2} \tilde{C}(x, t)}{d x^{2}}-k_{d} \tilde{C}(x, t)=0$.

Equation 4 is not a partial differential equation anymore but a second order ordinary differential equation which has only one variable $x$. Equation 4 indicates that the steady state distribution of signaling molecules show an exponential decay from the production site as

$\tilde{C}(x, t)=C_{0} e^{-x / \lambda}$,

$\lambda=\sqrt{\frac{D}{k_{d}}}$.

$C_{0}$ is the concentration at the left boundary, and $\lambda$ is the characteristic length at which the concentration decreases by a factor of $e$, namely, $\tilde{C}\left(x_{0}+\lambda, t\right)=\frac{1}{e} \tilde{C}\left(x_{0}, t\right)$ for any $x_{0}$ ( $e$ is the base of the natural logarithm, $\sim 2.7$ ). It is straight forward to check that Eq. 5 combined with Eq. 6 is the solution of Eq. 4, and that this solution obviously satisfies the boundary condition at $x=\infty$ (Eq. 3). We can determine $C_{0}$ so that the concentration profile also satisfies the boundary condition at $x=0$. By using Eqs. 2 and $5, C_{0}$ is determined as follows

$C_{0}=\frac{J}{\sqrt{D \cdot k_{d}}}$.

Note that we here neglect the effects of cell movement/ growth/division on the gradient formation, and treat that transportation of signaling molecules rely on only movement between cells. Caution is required here that recent studies reported a relationship between morphogen distribution and tissue growth, suggesting that this assumption is not always validated (Averbukh et al. 2014; Wartlick et al. 2014).

Such an exponential gradient of signaling molecules has been observed in several developmental systems, including Bicoid in Drosophila syncytial $(\lambda=80-100 \mu \mathrm{m})$ (Gregor et al. 2005; Houchmandzadeh et al. 2002); Decapentaplegic (Dpp) and Wingless in Drosophila wing disks $(\lambda=20$ and $6 \mu \mathrm{m}$, respectively) (Kicheva et al. 2007); and Fibroblast growth factor 8 in a zebrafish gastrulating embryo $(\lambda=200 \mu \mathrm{m})$ (Yu et al. 2009). These observations indicate that the in vivo movement of signaling molecules can be described as a diffusion process.

Equations 5, 6 and 7 show how signaling molecule mobility determines their steady-state distribution. When the signaling molecules move faster (namely, the diffusion coefficient $D$ is larger), the characteristic length $\lambda$ increases, resulting in a broader distribution of signaling molecules (Fig. 1b). In contrast, we can see a steeper distribution of signaling molecules when $\lambda$ is smaller due to slower movement (Fig. 1b). The degradation rate of signaling molecules, $k_{\mathrm{d}}$, also affects the shape of the gradient; this is well described in literatures (Dorocco et al. 2011; Inomata et al. 2008, 2013). These studies reported that perturbation on protein stability influences the distribution pattern of morphogen, in consistent with the idea in SDD model (Dorocco et al. 2011; Inomata et al. 2008, 2013). Such perturbation experiments are helpful to know the mechanism for morphogen gradient formation, however, to the best of our knowledge, experiment in which diffusion coefficient of morphogen is artificially changed has not yet been reported.

Quantitative investigation of signaling molecule mobility

Mobility of signaling molecules can be investigated quantitatively by the FRAP assay. In a FRAP experiment, 
fluorescently-labeled morphogens in a region of interest are irreversibly photobleached by a strong laser, followed by measurement of fluorescence recovery over time. The curve of fluorescence recovery contains information on the mobility of the molecule analyzed. For example, Dpp fused with green fluorescent protein (GFP) in a Drosophila wing imaginal disk has a diffusion coefficient $D=0.1 \pm 0.05 \mu \mathrm{m}^{2} \mathrm{~s}^{-1}$ (Kicheva et al. 2007), which is 1000 -fold smaller than GFP in water $\left(D=\sim 100 \mu \mathrm{m}^{2} / \mathrm{s}\right.$, Terry et al. 1995). Small mobility mediated by non-directed transcytosis involving recurrent cellular uptake and release is proposed to be a key event in the formation of a Dpp gradient (Kicheva et al. 2007).

It is difficult to fully elucidate the mechanism controlling the mobility of signaling molecules because of complex underlying processes including extracellular diffusion, cellular uptake, transcytosis, cell movement and direct delivery via cytonemes (cellular projections of animal cells) (Hsiung et al. 2005; Kornberg 2012; Mülle et al. 2013; Pfeiffer et al. 2000; Ramirez-Weber and Kornberg 1999; Zhou et al. 2012). Indeed, a recent study found that the diffusion coefficient of GFP-Dpp increases tenfold when analyzing the FRAP data by the SDD model with slight modification, taking into consideration diffusion in the extracellular matrix and cellular uptake (Zhou et al. 2012). The tissue geometry with membrane invaginations can make the diffusion process anomalous (Daniels et al. 2012), suggesting that mobility of signalling molecule varies depending on also spatial scales (Abu-Arish et al. 2010; Castle et al. 2011; Gregor et al. 2007).

We have seen that mobility of signaling molecules can determine the characteristic distribution on tissue scale, and that the mobility has been determined in several animal systems. It is no doubt that these studies pioneer a new field to investigate the mechanisms that mediates the morphogen gradient formation, however, the mechanism is not yet fully characterized. The time scale of the FRAP recovery curve is not simply related to that of signaling molecules' mobility, and measurements at different spatial scales often cause fluctuation of the diffusion coefficient. These problems arise from the complex transportation mechanism of signaling molecules and the tissue geometry in animal development. To explain the distribution of signaling molecule, simple experimental systems are required. In the latter part of this review, we will highlight the potential advantages of plant developmental systems.

Plasmodesmata, a unique structure in a plant cell

Plant developmental systems have some potential advantages to quantitatively assess the intercellular signaling dynamics with which we can overcome the difficulties described above. A notable structure of plant cells in terms of intercellular signaling is the plasmodesmata, which provides cytoplasmic continuity between neighboring cells (reviewed in Brunkard et al. 2013; Burch-Smith and Zambryski 2012; Xu and Jackson 2010). Some nutrients, such as sucrose, RNAs and proteins, can pass through the plasmodesmata, facilitating communication between cells. This direct connection between plant cells means that the effects of cell movement on the distribution of signaling molecules are negligible in plant development, namely plant organogenesis takes place without cell movement. This plant-specific architecture is highly dynamic during development. The plasmodesmata forms a simple structure in young emerged leaves, whereas it becomes more complex in older leaves (Fitzgibbon et al. 2013; Oparka et al. 1999). Protein mobility is also altered in association with this structural change (Oparka et al. 1999), hence regulation of the number and structure of plasmodesmata are critical mechanisms for plant organogenesis via intercellular signaling.

The influences of the aperture size of plasmodesmata on the mobility of signaling molecules have been experimentally examined by monitoring movement of fluorescent probes between plant cells, and been mathematically investigated (Dölger et al. 2014; Liesche and Schulz 2013; Terry and Robards 1987). To estimate the influence of the simple plasmodesmata on the mobility of signaling molecules, first, we consider a situation that the dwell time of the particle in plasmodesmata is shorter than the characteristic time scale of intercellular diffusion. Under this situation, diffusion coefficient at the cell boundary is the same as the permeability across the boundary (Paine et al. 1975). If the aperture is infinitely large, the permeability of signaling molecule is equal to the diffusion coefficient in cytoplasm, which we call $D_{0}$. If the aperture is smaller than size of a molecule, there is no transportation, meaning that the permeability is zero. To consider the effects of finite aperture size on the permeability, we model a signaling molecule as a sphere with a radius $a$, and plasmodesmata as a hollow cylinder with a radius $r$. There are two hindrance effects of plasmodesmata that reduce the permeability: (1) molecules hitting the aperture edge are reflected and does not enter into the plasmodesmata, and (2) effective viscosity increases due to the presence of plasmodesmata wall. (1) is purely geometrical effect and is calculated as a probability $F_{1}$ that an particle approaching to an aperture does not hit the edge,

$F_{1}=\frac{\pi(r-a)^{2}}{\pi r^{2}}=\left(1-\frac{a}{r}\right)^{2}$.

Second factor $F_{2}$ is more complicated, however, can be estimated as an increase of hydrodynamic drag force on a particle moving in a center of a cylinder filled with stationary Newtonian fluid (Pain and Scherr 1975). In this case, $F_{2}$ is approximately given as 
$F_{2} \sim \frac{1-0.75857\left(a_{/ r}\right)^{5}}{1-2.1050(a / r)+2.0865\left(a_{/}\right)^{3}-1.7068\left(a_{/ r}\right)^{5}+0.72603\left(a_{/ r}\right)^{6}}$

(Haberman and Sayre 1958). Taken together, the effects of plasmodesmata aperture on diffusion coefficient is

$$
\begin{aligned}
\frac{D}{D_{0}}= & F_{1} \times F_{2}=\left(1-\frac{a}{r}\right)^{2} \\
& \times \frac{1-0.75857(a / r)^{5}}{1-2.1050(a / r)+2.0865(a / r)^{3}-1.7068\left(a_{r}\right)^{5}+0.72603\left(a_{r}\right)^{6}} .
\end{aligned}
$$

Using Eq. 10, we plotted the normalized diffusion coefficient $D / D_{0}$ as a function of aperture size $r$ with numerically obtained $F_{2}$ (Pain and Scherr 1975) (Fig. 2). Here we used the radius of GFP $(\sim 2 \mathrm{~nm})$ as the size of signaling molecule (Ormö et al. 1996; Pack et al. 2006). Interestingly, the protein mobility is largely affected even when an aperture size is ten times larger than the protein size, and can be changed by more than two folds by modifying aperture radius from 10 to $40 \mathrm{~nm}$.

Deposition and degradation of callose at the neck of the plasmodesmata largely determine the aperture size and therefore also the movement of signaling molecules (Reviewed in Brunkard et al. 2013; De Storme and Geelen 2014). By modulating the expression levels and pattern of callose synthases and $\beta$-1, 3-glucanases, which are involved in callose turnover (e.g. Guseman et al. 2010; Levy et al. 2007), the conductivity can be altered in a tissue-specific manner (Vatén et al. 2011). This technique enabled us to conduct a perturbation experiment on the mobility of

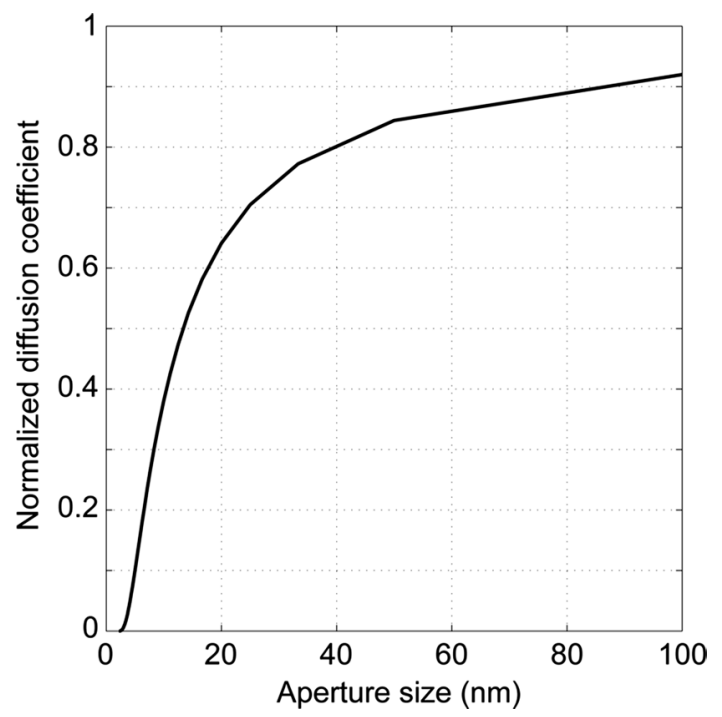

Fig. 2 Relationship between aperture size of plasmodesmata and molecular mobility. Normalized diffusion coefficient of a molecule ( $2 \mathrm{~nm}$ radius) is plotted as a function of radius size of aperture using Eq. 10 signaling molecules (Vatén et al. 2011), and facilitated understanding of the effect of signaling molecule mobility on the formation of its characteristic distribution. As for the transportation of signaling molecules via plasmodesmata, it was also revealed recently that a chaperone complex facilitates protein movement via the plasmodesmata, possibly through protein unfolding and refolding (Xu et al. 2011).

As we have seen, movement of signaling molecules via the plasmodesmata could be used to quantitatively investigate the mobility of signaling molecules. In the next section, we will introduce two non-cell-autonomous signaling molecules, AN3 and UPBEAT1 (UPB1), which exhibit interesting behaviour in plant leaves and roots, respectively (Kawade et al. 2013; Tsukagoshi et al. 2010).

Molecular mechanism of intercellular signaling in plantsAN3 and UPB1

The transition from cell proliferation to post-mitotic cell expansion marks the initial step in cellular differentiation. Spatiotemporal control of this transition within a developing organ is a quintessential feature of organogenesis. In Arabidopsis, mitotic cells are distributed uniformly in young leaf primordia. The proliferation phase is then arrested from the leaf tip to the base region, followed by the beginning of the post-mitotic phase (Andriankaja et al. 2012; Donnelly et al. 1999; Kazama et al. 2010). Dynamics of the boundary of this transition during leaf development have been well described (Andriankaja et al. 2012; Donnelly et al. 1999; Kazama et al. 2010; Nath et al. 2003; Tsukaya 2014; White 2006). The CINCINNATA (CIN) in Antirrhinum and TCP-family genes in Arabidopsis form a subclade of the class II TCPs (CIN-TCPs) and are involved in the progression of the boundary (Efroni et al. 2008; Nath et al. 2003). The SWITCH/SUCROSE NONFERMENTING (SWI/SNF) chromatin remodelling ATPase BRAHMA (BRM) was recently shown to interact with CIN-TCPs to regulate the expression of their downstream genes, and then control boundary progression (Efroni et al. 2013). In addition to CIN-TCPs, also AN3 is recruited into the SWI/SNF complex for active proliferation of leaf cells (Vercruyssen et al. 2014). The SWI/SNF-TCPs and -AN3 mediated regulation is, therefore, proposed to explain, at a molecular level, how the boundary between cell proliferation and post-mitotic cell expansion is spatiotemporally determined during leaf development. Although this idea is appealing, cell proliferation occurs in a broader area than does expression of AN3 (Fig. 3; Horiguchi et al. 2005). Because AN3 can move between leaf epidermal and mesophyll cells (Kawade et al. 2013), one possible explanation for the spatial difference is that the AN3 protein moves within the same cell layer. It would be interesting to determine whether the spatiotemporal dynamics of AN3 
distribution corresponds to the transition from cellular proliferation to differentiation during leaf development. This is to be discussed in the next chapter.

Our knowledge, at the molecular level, of the definition of the boundary between cell proliferation and cell elongation where cellular differentiation begins in roots is more advanced (Breuer et al. 2010; Dello Ioio et al. 2007). A basic helix-loop-helix domain (bHLH) transcription factor known as UPBEAT1 (UPB1) is one of the key components in this process (Tsukagoshi et al. 2010). The UPBI promoter is active in cells of the lateral root cap (LRC) near the transition zone and in vascular cells of the differentiation zone (Tsukagoshi et al. 2010). In contrast, when UPB1, fused with GFP, is expressed under the control of the same promoter, GFP signal is observed in all cells in the differentiation zone, and also weakly in the cells of the proliferation zone, perhaps due to the movement of UPB 1 (Tsukagoshi et al. 2010). These results, together with the data from a series of transgenic lines expressing UPB1 fused with GFP or with tandem three YFPs, suggest that UPB1 is a signaling molecule originating from the LRC that regulates the transition between cell proliferation and differentiation in roots (Tsukagoshi et al. 2010). Transcriptome, ChIP-chip and chemical treatment experiments have revealed that UPB1 directly controls the expression of peroxidases to modulate the balance of reactive oxygen species (ROS) (Tsukagoshi et al. 2010). Although ROS metabolism is known to be involved in the control of the balance between cell proliferation and differentiation in plants and animals (Ostrakhovitch and Semenikhin 2013; Wang et al. 2013) the underlying mechanism remains to be elucidated.

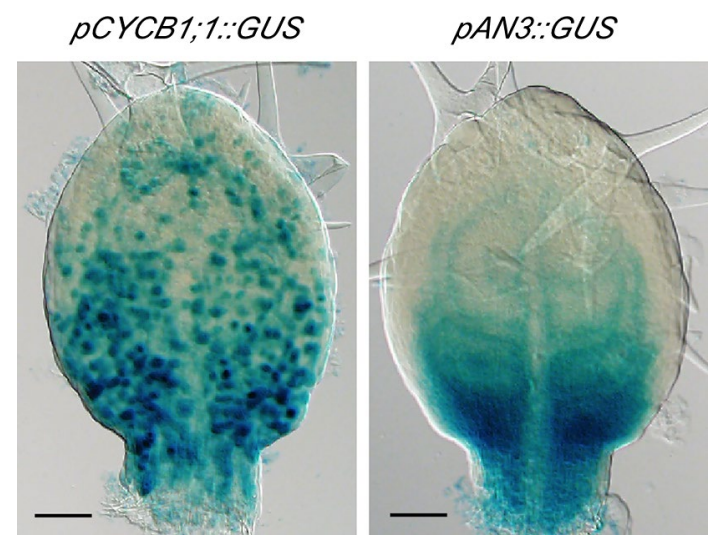

Fig. 3 Distribution of proliferating cells and expression pattern of $A N 3$ in the first leaf primordia. A cyclin-B1;1 promoter- $\beta$ glucuronidase (GUS) reporter line ( $p C Y C B 1 ; 1:: G U S$, Donnelly et al. 1999) and an AN3 promoter-GUS reporter line (pAN3::GUS, Horiguchi et al. 2005) were used to visualize proliferating cells and AN3-expressing cells, respectively. Leaf primordia are from 6-day-old seedlings. Method for GUS staining is described elsewhere (Kawade et al. 2013). Bars $50 \mu \mathrm{m}$
Identification of UPB1 signaling would stimulate further study of the mechanism linking ROS homeostasis with the transition from cell proliferation to differentiation via intercellular signaling.

Toward quantitative understanding of intercellular signaling in plant organogenesis

Our understanding of the functions of AN3 and UPB1 at the molecular level is increasing, but quantitative data regarding their mobility are lacking. This is also true for other signaling molecules that play an important role in plant development. Whether AN3 forms an expression gradient along the leaf proximal-to-distal axis, related to cell proliferation activity, is unknown. If this is the case, the underlying mechanism via intercellular movement is of great interest. Quantitative characterisation of AN3 signaling, together with theoretical investigation based on the SDD model, is thus essential to determine whether AN3 movement occurs in a non-directional manner, whether AN3 movement between cells is mediated simply by diffusion, whether diffusion is sufficient to explain AN3 distribution, and whether the kinetics of spatiotemporal dynamics of cell proliferation are consistent with the AN3signaling dynamics. To address these issues, some essential parameters could experimentally be determined through FRAP assays where the size of the region of bleaching is modified from single cell to tissue scales (Kawade et al. unpublished): when we photobleach at single cell level, diffusion coefficient and mode of diffusivity between individual cells can be characterized. We can assess directionality and tissue-scale kinetics of protein mobility by analyzing FRAP data with larger region for bleaching, for instance, we may observe direction-dependent signal recovery, which is a sign of directional transportation. The intracellular mobile fraction of AN3 is also interesting, as AN3 is localised mainly to the nucleus and, to a lesser extent, the cytoplasm (Kawade et al. 2010, 2013). Recovery curve of FRAP assay at tissue scale include intermixed information about intracellular and intercellular mobility of signaling molecule (Sprague and McNally 2005). Together, to precisely know the AN3-signaling dynamics within a developing organ, we have to distinguish these mobility kinetics by comparing data from single-cell and tissue-scale FRAP assays. AN3-signaling dynamics can be recapitulated by integrating these parameters in SDD model for further investigation of relationship to cell proliferation dynamics. Similar questions are relevant in the case of UPB1. Such information would clarify the mechanism underlying the control of plant organogenesis by intercellular signaling via the plasmodesmata.

Quantitative studies using plant materials could facilitate investigation of the signaling dynamics during plant 
organogenesis. A transmembrane leucine-rich-repeat receptor-like kinase FLAGELLIN SENSITIVE2 (FLS2) is a component of defence signaling against plant pathogens (Gomez-Gomez and Boller 2000). FRAP assay revealed that $75 \%$ of the FLS2 in the plasma membrane diffuse laterally, with $D=0.34 \pm 0.02 \mu \mathrm{m}^{2} \mathrm{~s}^{-1}$ (Ali et al. 2007). However, FLS2 transport is slower, with $D=0.22 \pm 0.01 \mu \mathrm{m}^{2} \mathrm{~s}^{-1}$, in the presence of flagellin of plant pathogenic bacteria (Ali et al. 2007). This fact suggests that FLS2 signaling is modulated by a change in its mobility in response to bacterial infection. In addition to determine the diffusion coefficient, the FRAP assay is helpful in understanding the mobile intracellular fraction and directionality. Murata et al. (2013) investigated microtubule behaviour during phragmoplast expansion, using FRAP and other imaging techniques, and revealed two types of microtubules, the stable and dynamic. Kitagawa and Fujita (2013) explored protein diffusivity in protonema cells of Physcomitrella patens using the photoconversion protein Dendra2 and demonstrated that protein diffusivity in these cells exhibited directional bias. The utility of Dendra2 is confirmed also in Arabidopsis (Wu et al. 2011).

Above-mentioned studies focused mainly on protein diffusivity at a small scale (local diffusivity) (Ali et al. 2007; Kitagawa and Fujita 2013; Murata et al. 2013). Our next challenge is to assess the dynamics of signaling molecules at a larger scale (global diffusivity) to explain the mechanisms by which the tissue-scale distribution of signaling molecules is established during organogenesis, and its control of developmental progression. The wealth of quantitative imaging techniques, together with a unique mode of intercellular signaling in plants, will allow us to decipher the puzzle of organogenesis via intercellular signaling.

Acknowledgments We are grateful to Drs. Tomomichi Fujita (Hokkaido University, Japan), Masami Yokota Hirai (RIKEN CSRS, Japan) and Nicolas Minc (Institut Jacques Monod, France) for giving us an opportunity to freely contribute to this work. This work was supported by fellowships from the RIKEN Special Postdoctoral Researchers Program (No. K23253 to KK) and the Japan Society for the Promotion of Science (JSPS) (No. 262337 to KK).

\section{References}

Abu-Arish A, Porcher A, Czerwonka A, Dostatni N, Fradin C (2010) High mobility of Bicoid captured by fluorescence correlation spectroscopy: implication for the rapid establishment of its gradient. Biophys J 99:33-35

Ali GS, Prasad KV, Day I, Reddy AS (2007) Ligand-dependent reduction in the membrane mobility of FLAGELLIN SENSITIVE2, an Arabidopsis receptor-like kinase. Plant Cell Physiol 48:1601-1611

Andriankaja M et al (2012) Exit from proliferation during leaf development in Arabidopsis thaliana: a not-so-gradual process. Dev Cell 22:64-78
Averbukh I, Ben-Zvi D, Mishra S, Barkai N (2014) Scaling morphogen gradients during tissue growth by a cell division rule. Development 141:2150-2156

Berezhkovskii AM, Sample C, Shvartsman SY (2010) How long does it take to establish a morphogen gradient? Biophys J 99:59-61

Berg HC (1993) Random walks in biology. Princeton University Press, UK

Bergmann S, Sandler O, Sberro H, Shnider S, Schejter E, Shilo BZ, Barkai N (2007) Pre-steady-state decoding of the Bicoid morphogen gradient. PLoS Biol 5:e46

Breuer C, Ishida T, Sugimoto K (2010) Developmental control of endocycles and cell growth in plants. Curr Opin Plant Biol 13:654-660

Brown R (1828) A brief account of microscopical observations made in the months of June, July, and August, 1827, on the particles contained in the pollen of plants; and on the general existence of active molecules in organic and inorganic bodies. Edinb N Philos J 358-371

Brunkard JO, Runkel AM, Zambryski PC (2013) Plasmodesmata dynamics are coordinated by intracellular signaling pathways. Curr Opin Plant Biol 16:614-620

Burch-Smith TM, Zambryski PC (2012) Plasmodesmata paradigm shift: regulation from without versus within. Annu Rev Plant Biol 63:239-260

Castle BT, Howard SA, Odde DJ (2011) Assessment of transport mechanisms underlying the Bicoid morphogen gradient. Cell Mol Bioeng 4:116-121

Crick F (1970) Diffusion in embryogenesis. Nature 225:420-422

Daniels BR, Rikhy R, Renz M, Dobrowsky TM, Lippincott-Schwartz J (2012) Multiscale diffusion in the mitotic Drosophila melanogaster syncytial blastoderm. Proc Natl Acad Sci USA 109:8588-8593

De Storme N, Geelen D (2014) Callose homeostasis at plasmodesmata: molecular regulators and developmental relevance. Front Plant Sci 5:138

Dello Ioio R, Linhares FS, Scacchi E, Casamitjana-Martinez E, Heidstra R, Costantino P, Sabatini S (2007) Cytokinins determine Arabidopsis root-meristem size by controlling cell differentiation. Curr Biol 17:678-682

Dölger J, Rademaker H, Liesche J, Schulz A, Bohr T (2014) Diffusion and bulk flow in phloem loading: a theoretical analysis of the polymer trap mechanism for sugar transport in plants. Phys Rev E 90:042704

Donnelly PM, Bonetta D, Tsukaya H, Dengler RE, Dengler NG (1999) Cell cycling and cell enlargement in developing leaves of Arabidopsis. Dev Biol 215:407-419

Dorocco JA, Grimm O, Tank DW, Wieschaus E (2011) Measurement and perturbation of morphogen lifetime: effects on gradient shape. Biophys J 101:1807-1815

Efroni I, Blum E, Goldshmidt A, Eshed Y (2008) A protracted and dynamic maturation schedule underlies Arabidopsis leaf development. Plant Cell 20:2293-2306

Efroni I et al (2013) Regulation of leaf maturation by chromatin-mediated modulation of cytokinin responses. Dev Cell 24:438-445

Fitzgibbon J, Beck M, Zhou J, Faulkner C, Robatzek S, Oparka K (2013) A developmental framework for complex plasmodesmata formation revealed by large-scale imaging of the Arabidopsis leaf epidermis. Plant Cell 25:57-70

Gomez-Gomez L, Boller T (2000) FLS2: an LRR receptor-like kinase involved in the perception of the bacterial elicitor flagellin in Arabidopsis. Mol Cell 5:1003-1011

Gregor T, Bialek W, de Ruyter van Steveninck RR, Tank DW, Wieschaus EF (2005) Diffusion and scaling during early embryonic pattern formation. Proc Natl Acad Sci USA 102:18403-18407

Gregor T, Wieschaus EF, McGregor AP, Bialek W, Tank D (2007) Stability and nuclear dynamics of the Bicoid morphogen gradient. Cell 130:141-152 
Guseman JM et al (2010) Dysregulation of cell-to-cell connectivity and stomatal patterning by loss-of-function mutation in Arabidopsis chorus (glucan synthase-like 8). Development 137:1731-1741

Haberman WL, Sayre RM (1958) Motion of rigid and fluid spheres in stationary and moving liquids inside cylindrical tubes. David Taylor Model Basin, Report 1143

Horiguchi G, Kim GT, Tsukaya H (2005) The transcription factor AtGRF5 and the transcription coactivator AN3 regulate cell proliferation in leaf primordia of Arabidopsis thaliana. Plant $\mathrm{J}$ 43:68-78

Houchmandzadeh B, Wieschaus E, Leibler S (2002) Establishment of developmental precision and proportions in the early Drosophila embryo. Nature 415:798-802

Hsiung F, Ramirez-Weber FA, Iwaki DD, Kornberg TB (2005) Dependence of Drosophila wing imaginal disc cytonemes on Decapentaplegic. Nature 437:560-563

Inomata H, Haraguchi T, Sasai Y (2008) Robust stability of the embryonic axial pattern requires a secreted scaffold for Chordin degradation. Cell 134:854-865

Inomata H, Shibata T, Haraguchi T, Sasai Y (2013) Scaling of dorsalventral patterning by embryo size-dependent degradation of Spemann's organizer signals. Cell 153:1296-1311

Jackson D, Veit B, Hake S (1994) Expression of maize KNOTTED1 related homeobox genes in the shoot apical meristem predicts patterns of morphogenesis in the vegetative shoot. Development 120:405-413

Kawade K, Horiguchi G, Tsukaya H (2010) Non-cell-autonomously coordinated organ size regulation in leaf development. Development 137:4221-4227

Kawade K, Horiguchi G, Usami T, Hirai MY, Tsukaya H (2013) ANGUSTIFOLIA3 signaling coordinates proliferation between clonally distinct cells in leaves. Curr Biol 23:788-792

Kazama T, Ichihashi Y, Murata S, Tsukaya H (2010) The mechanism of cell cycle arrest front progression explained by a KLUH/ CYP78A5-dependent mobile growth factor in developing leaves of Arabidopsis thaliana. Plant Cell Physiol 51:1046-1054

Kicheva A, Pantazis P, Bollenbach T, Kalaidzidis Y, Bittig T, Jülicher F, Gonzalez-Gaitan M (2007) Kinetics of morphogen gradient formation. Science 315:521-525

Kicheva A, Bollenbach T, Wartlick O, Jülicher F, Gonzalez-Gaitan M (2012) Investigating the principles of morphogen gradient formation: from tissues to cells. Curr Opin Genet Dev 22:527-532

Kim JH, Kende H (2004) A transcriptional coactivator, AtGIF1, is involved in regulating leaf growth and morphology in Arabidopsis. Proc Natl Acad Sci USA 101:13374-13379

Kim JY, Yuan Z, Jackson D (2003) Developmental regulation and significance of KNOX protein trafficking in Arabidopsis. Development 130:4351-4362

Kitagawa M, Fujita T (2013) Quantitative imaging of directional transport through plasmodesmata in moss protonemata via single-cell photoconversion of Dendra2. J Plant Res 126: $577-585$

Kornberg TB (2012) The imperatives of context and contour for morphogen dispersion. Biophys J 103:2252-2256

Levy A, Erlanger M, Rosenthal M, Epel BL (2007) A plasmodesmataassociated $\beta$-1,3-glucanase in Arabidopsis. Plant J 49:669-682

Liesche J, Schulz A (2013) Modeling the parameters for plasmodesmatal sugar filtering in active symplasmic phloem loaders. Front Plant Sci 4:207

Mülle P, Rogers KW, Jordan BM, Lee JS, Robson D, Ramanathan S, Schier AF (2012) Differential diffusivity of Nodal and Lefty underlies a reaction-diffusion patterning system. Science 336:721-724

Mülle P, Rogers KW, Yu SR, Brand M, Schier AF (2013) Morphogen transport. Development 140:1621-1638
Murata T et al (2013) Mechanism of microtubule array expansion in the cytokinetic phragmoplast. Nat Commun 4:1967

Nakajima K, Sena G, Nawy T, Benfey PN (2001) Intercellular movement of the putative transcription factor SHR in root patterning. Nature 413:307-311

Nath U, Crawford BC, Carpenter R, Coen E (2003) Genetic control of surface curvature. Science 299:1404-1407

Oparka KJ et al (1999) Simple, but not branched, plasmodesmata allow the nonspecific trafficking of proteins in developing tobacco leaves. Cell 97:743-754

Ormö M, Cubitt AB, Kallio K, Gross LA, Tsien RY, Remington SJ (1996) Crystal structure of the Aequorea victoria green fluorescent protein. Science 273:1392-1395

Ostrakhovitch EA, Semenikhin OA (2013) The role of redox environment in neurogenic development. Arch Biochem Biophys 534:44-54

Pack C, Saito K, Tamura M, Kinjo M (2006) Microenvironment and effect of energy depletion in the nucleus analyzed by mobility of multiple oligometric EGFPs. Biophys J 91:3921-3936

Pain PL, Scherr P (1975) Drag coefficients for the movement of rigid spheres through liquid-filled cylindrical pores. Biophys $\mathbf{J}$ 15:1087-1091

Paine PL, Moore LC, Horowitz SB (1975) Nuclear envelope permeability. Nature 254:109-114

Pfeiffer S, Alexandre C, Calleja M, Vincent JP (2000) The progeny of wingless-expressing cells deliver the signal at a distance in Drosophila embryos. Curr Biol 10:321-324

Ramirez-Weber FA, Kornberg TB (1999) Cytonemes: cellular processes that project to the principal signaling center in Drosophila imaginal discs. Cell 97:599-607

Sprague BL, McNally JG (2005) FRAP analysis of binding: proper and fitting. Trends Cell Biol 15:84-91

Terry BR, Robards AW (1987) Hydrodynamic radius alone governs the mobility of molecules through plasmodesmata. Planta 171:145-157

Terry BR, Matthews EK, Haseloff J (1995) Molecular characterisation of recombinant green fluorescent protein by fluorescence correlation microscopy. Biochem Biophys Res Commun 217:21-27

Tsukagoshi H, Busch W, Benfey PN (2010) Transcriptional regulation of ROS controls transition from proliferation to differentiation in the root. Cell 143:606-616

Tsukaya H (2014) Comparative leaf development in angiosperms. Curr Opin Plant Biol 17:103-109

Turing A (1952) The chemical basis of morphogenesis. Phil Trans R Soc B 237:37-72

Vatén A et al (2011) Callose biosynthesis regulates symplastic trafficking during root development. Dev Cell 21:1144-1155

Vercruyssen L et al (2014) ANGUSTIFOLIA3 binds to SWI/SNF chromatin remodeling complexes to regulate transcription during Arabidopsis leaf development. Plant Cell 26:210-229

Wang K, Zhang T, Dong Q, Nice EC, Huang C, Wei Y (2013) Redox homeostasis: the linchpin in stem cell self-renewal and differentiation. Cell Death Dis 4:e537

Wartlick O, Kicheva A, Gonzalez-Gaitan M (2009) Morphogen gradient formation. Cold Spring Harb Perspect Biol 1:a001255

Wartlick O, Jülicher F, Gonzalez-Gaitan M (2014) Growth control by a moving morphogen gradient during Drosophila eye development. Development 141:1884-1893

White DW (2006) PEAPOD regulates lamina size and curvature in Arabidopsis. Proc Natl Acad Sci USA 103:13238-13243

Wolpert L (1969) Positional information and the spatial pattern of cellular differentiation. J Theor Biol 25:1-47

Wu S, Koizumi K, MacRae-Crerar A, Gallagher KL (2011) Assessing the utility of photoswitchable fluorescent proteins for tracking intercellular protein movement in the Arabidopsis root. PLoS One 6:e27536 
Xu XM, Jackson D (2010) Lights at the end of the tunnel: new views of plasmodesmal structure and function. Curr Opin Plant Biol 13:684-692

$\mathrm{Xu} \mathrm{XM}$ et al (2011) Chaperonins facilitate KNOTTED1 cell-to-cell trafficking and stem cell function. Science 333:1141-1144
Yu SR et al (2009) Fgf8 morphogen gradient forms by a source-sink mechanism with freely diffusing molecules. Nature 461:533-536 Zhou S, Lo WC, Suhalim JL, Digman MA, Gratton E, Nie Q, Lander AD (2012) Free extracellular diffusion creates the Dpp morphogen gradient of the Drosophila wing disc. Curr Biol 22:668-675 